\title{
Tuning the Aggregation of Titanate Nanowires in Aqueous Dispersions
}

\author{
Tamás Szabó, ${ }^{\dagger \neq}$ Viktor Tóth, ${ }^{\dagger}$ Endre Horváth, ${ }^{\S}$ László Forró, ${ }^{\S}$ and Istvan Szilagyi* ${ }^{\dagger}$ \\ ${ }^{\dagger}$ Department of Inorganic and Analytical Chemistry, University of Geneva, 1205 Geneva, Switzerland \\ ${ }^{\ddagger}$ Department of Physical Chemistry and Materials Science, University of Szeged, 6720 Szeged, Hungary \\ ${ }^{\S}$ Laboratory of Physics of Complex Matter, École Polytechnique Fédérale de Lausanne, 1015 Lausanne, Switzerland
}

\section{Supporting Information}

\begin{abstract}
Electrophoretic and dynamic light scattering (DLS) measurements revealed that aggregation in aqueous dispersion of titanate nanowires (TiONWs) can be tuned by poly(diallyldimethylammonium) chloride (PDADMAC) polyelectrolyte. The nanowires possessed negative charge under alkaline conditions which was compensated by the oppositely charged PDADMAC adsorbed on the surface. Such adsorption led to charge neutralization and subsequent charge reversal at the appropriate polyelectrolyte doses. The dispersions were stable at low PDADMAC concentration where the TiONWs possessed negative charge. However, fast aggregation of the nanowires occurred close to the charge neutralization point where the overall charge of the particles was zero. Charge inversion at high polyelectrolyte doses gave rise to restabilization of the samples and slow aggregation of the TiONWs even at higher ionic strengths where the original bare TiONW dispersions were unstable. The colloid stability of the bare nanowires can be explained well qualitatively by the Derjaguin, Landau, Verwey, and Overbeek (DLVO) theory; however, polyelectrolyte adsorption led to additional patch-charge attractions and osmotic repulsion between the particles. On the basis of the knowledge generated by the present work, experimental conditions (e.g., salt level, polyelectrolyte, and particle concentrations) can be adjusted in order to design stable and processable aqueous dispersions of TiONWs for further applications.
\end{abstract}

\section{INTRODUCTION}

One of the most frequently investigated titanate derivatives nowadays are the titanate nanowires (TiONWs) due to the growing number of their applications for instance in sensing as materials for high-sensitivity accelerometer ${ }^{1}$ or humidity sensor, ${ }^{2}$ in electrochemistry as electrode constituents, ${ }^{3,4}$ in solar cells as photoanode, and in catalysis. For the latter, similarly to other titanium(IV) compounds, ${ }^{5-7}$ they have been used as catalysts in photocatalytic decomposition of various organic molecules ${ }^{8,9}$ as well as catalyst support in hydrogenation reactions. ${ }^{10}$ Many of their unique properties (e.g., metal-induced transformation into another form at high temperature $^{11}$ and surface conductance changes due to adsorption of small molecules ${ }^{12}$ ) related to these applications have been investigated in detail, but the majority of these studies contained investigations regarding their behavior only in the solid state. However, aqueous colloid dispersions of TiONWs are widely used during synthesis processes for these applications, and their colloid stability has to be tuned to obtain the desired dispersion properties. Accordingly, suspended TiONWs can be removed from the liquid medium by aggregation, subsequent sedimentation, and/or filtration, whereas a long-term kinetic stability of ultrafine dispersions of primary particles representing a high specific surface area can be achieved by preventing the aggregation with a stabilizing agent. In spite of the importance of the stability of these disperse systems, only a limited number of related studies are available, and there is a lack of quantitative description of the charging and aggregation processes of titanate nanowires in the literature. ${ }^{13,14}$

It is well-known that charged polymers or so-called polyelectrolytes adsorb strongly on oppositely charged surfaces, ${ }^{15-17}$ and they have been widely used to adjust aggregation processes in dispersions of charged particles. ${ }^{18-22}$ Adsorption properties have been also utilized to construct hybrid materials containing titanium(IV) compounds and polyelectrolytes $^{23,24}$ as well as to control aggregation in aqueous dispersions of barium titanates, ${ }^{25,26}$ lead zirconate titanates, ${ }^{27}$ titanate nanotubes, ${ }^{28}$ and titania nanoparticles. ${ }^{29}$ Among the polyelectrolytes, poly(diallyldimethylammonium) chloride (PDADMAC) has been applied for surface treatment or as coagulating agent in various systems and coating agent to create biofunctionalized nanoparticles for therapeutic applications. ${ }^{30}$ Accordingly, careful investigations performed with surface sensitive techniques such as reflectometry, ellipsometry, and atomic force microscopy as well as theoretical predictions revealed that PDADMAC adsorbs strongly on an oppositely charged surface, but the adsorption rate and amount greatly

Received: August 7, 2014

Revised: December 18, 2014

Published: December 19, 2014 
depend on the experimental conditions (e.g., concentration of the polyelectrolyte, ionic strength, and $\mathrm{pH}) .^{31,32}$ Electrophoretic mobility (EPM), streaming potential, and light scattering measurements revealed that PDADMAC adsorption results in charge neutralization and also charge reversal of an oppositely charged surface at appropriately high concentrations due to hydrophobic interactions, entropic effect, or ion-ion correlation forces. ${ }^{33-36}$ Such change in the sign of the surface charge is due to the formation of a polyelectrolyte layer with a thickness of a few nanometers depending on the experimental conditions applied..$^{2,37,38}$ Dynamic light scattering (DLS) studies pointed out that the hydrodynamic thickness of the PDADMAC layer adsorbed on colloidal latex particles increases with the ionic strength due to conformational changes of the adsorbed polyelectrolyte chains. At high salt levels, the electrostatic interactions between the adsorbed molecules and the electrolyte led to the formation of tails and loops extending away from the surface and hence to higher layer thickness values. ${ }^{33}$ Surface charge neutralization of colloidal particles with the oppositely charged PDADMAC gave rise to fast aggregation of the particles while the charge reversal phenomenon enabled stabilization of the system. ${ }^{34}$ Coating these particles with the polyelectrolyte significantly increased the stability of the dispersions. $^{22}$ Although, to the best of our knowledge, no studies have been reported on the interaction between TiONWs and PDADMAC, this polyelectrolyte was widely used in systems containing other titanate derivatives. Accordingly, titanate nanosheets were adsorbed on a PDADMAC modified surface to form perovskite-type thin films, ${ }^{39}$ multilayer films were obtained by the layer-by-layer technique using PDADMAC as a "glue" to strongly bind adjacent titania or titanate nanotubes, ${ }^{40,41}$ and a piezoelectric paper was fabricated by adsorbing barium titanate on wood cellulose fibers after surface modification with PDADMAC. ${ }^{42}$

Since TiONWs possess negative charge at $\mathrm{pH}$ values higher than their point of zero charge (PZC), ${ }^{13}$ the positively charged PDADMAC is a promising candidate to tune the colloid stability of the nanowire dispersions. The polyelectrolyte adsorption is expected to change the surface charge properties of the particles, and hence, aggregation processes can be adjusted by the addition of the PDADMAC. Accordingly, charging and aggregation of TiONWs in the presence of PDADMAC have been investigated by electrophoresis and DLS in the present work. The effects of PDADMAC dose, ionic strength, and TiONW concentration on the EPMs and speed of particle aggregation were clarified. The TiONWs have been characterized in the solid state by various experimental techniques previously; ${ }^{2,13,43}$ therefore, our study focused only on the dispersion properties. These findings will be the basis to design stable and processable biocompatible aqueous dispersions of TiONWs for further targeted applications such as thermal cancer therapy or drug delivery.

\section{EXPERIMENTAL SECTION}

Electrophoretic Mobility Measurements. The electrophoretic mobility (EPM) and time-resolved dynamic light scattering (DLS) measurements were performed with a ZetaNano ZS (Malvern Instruments) device equipped with a $\mathrm{He} / \mathrm{Ne}$ laser operating at 633 $\mathrm{nm}$ as a light source and an avalanche photodiode as a detector. A scattering angle of $173^{\circ}$ was applied. The device applies an electric field strength of $4 \mathrm{kV} / \mathrm{m}$ in the electrophoretic mode.

The EPM measurements were carried out in plastic capillary cells (Malvern Instruments) cleaned with 2 wt \% Hellmanex solution (Hellma) and rinsed extensively with Milli-Q water. For sample preparation, a calculated amount of PDADMAC solution was mixed with water and $\mathrm{KCl}$ stock solution to obtain $4.5 \mathrm{~mL}$ sample with the desired polyelectrolyte concentration and ionic strength. Thereafter, the procedure was finalized by adding $0.5 \mathrm{~mL}$ of about $70 \mathrm{mg} / \mathrm{L}$ TiONW dispersion to the samples in a plastic vial. Accordingly, the final particle concentration was always around $7 \mathrm{mg} / \mathrm{L}$, and the total sample volume was $5.0 \mathrm{~mL}$. The $\mathrm{pH}$ of the stock solutions was adjusted to 9 before mixing them. The EPMs were measured after equilibrating the dispersions overnight at room temperature and also for $1 \mathrm{~min}$ in the instrument prior to the measurement. The EPM $(\mu)$ of the samples was calculated by averaging five individual measurements. The surface charge density $(\sigma)$ of the bare and polyelectrolyte coated particles was determined by fitting the surface potential values at different ionic strengths with the linear charge-potential relation within the Debye-Hückel approximation ${ }^{44}$

$$
\sigma=\varepsilon \varepsilon_{0} \kappa \psi_{\mathrm{D}}
$$

where $\varepsilon \varepsilon_{0}$ is the permittivity of the water, $\psi_{\mathrm{D}}$ is the diffuse layer potential, and $\kappa$ is the inverse Debye length which can be calculated by taking the presence of the ionic species into account (see Supporting Information for more details). The $\psi_{\mathrm{D}}$ data were converted from the $\mu$ values using the Henry equation ${ }^{45}$

$$
\mu=f(x) \frac{\varepsilon \varepsilon_{0}}{\eta} \psi_{\mathrm{D}}
$$

where $f(x)$ is the Henry function which takes the Debye length and the size of the particles into consideration and $\eta$ is the viscosity of water.

Light Scattering. In the time-resolved DLS experiments, the apparent hydrodynamic radii $\left(r_{\mathrm{h}}\right)$ were determined using a secondorder cumulant fit for the correlation function which was accumulated for $30 \mathrm{~s}$ using the same device as for electrophoretic experiments. The measurements were carried out in $1 \mathrm{~cm}$ square plastic cuvettes (Malvern Instruments) cleaned by 2 wt \% Hellmanex solution. In the time-resolved DLS experiments, typically 50 runs were performed over $25 \mathrm{~min}$, and the samples were prepared as follows. Appropriate volumes of water, PDADMAC, and $\mathrm{KCl}$ stock solutions were mixed, which resulted in $1.8 \mathrm{~mL}$ solution with the desired polyelectrolyte concentration and ionic strength. The aggregation experiment was initiated by injecting $0.2 \mathrm{~mL}$ of the TiONW stock dispersion (which was previously subjected to ultrasonic treatment to minimize the initial aggregation) into the solutions. The final particle concentration was about $7 \mathrm{mg} / \mathrm{L}$, the total volume was $2 \mathrm{~mL}$, and the $\mathrm{pH}$ of all stock solutions was adjusted to 9 . Such low particle concentration allowed us to avoid multiple scattering events (Figure S1) and to remain in the early stages of the aggregation process indicated by single-exponential decays of the correlation function even at the end of the experiments (Figure S2). The slopes of the apparent hydrodynamic radii versus time curves were calculated in the linear regimes and normalized by the mass concentrations $(w)$. The colloid stability of the systems was expressed in terms of the stability ratio $(W)$ as

$$
W=\frac{S_{\text {fast }} w}{S w_{\text {fast }}}
$$

where $S_{\text {fast }}$ and $S$ are the slopes in the presence of an excess of indifferent electrolyte (1 M KCl, fast aggregation occurs) and for the actual measurements, respectively, and $w_{\text {fast }}$ and $w$ are the mass concentrations of the TiONW in the dispersions for fast aggregation (1 $\mathrm{M} \mathrm{KCl})$ and for the actual experiment, respectively. The aggregation of TiONWs was controlled only by the diffusion of the nanowires in the fast case in $1 \mathrm{M} \mathrm{KCl}$ solutions; therefore, stability ratios close to unity correspond to unstable systems while higher values refer to more stable samples.

In addition, combined static light scattering and DLS experiments performed on a multiangle goniometer revealed that the decay constants of the autocorrelation functions showed linear dependence of a zero intercept with the square of the scattering vector (Figure S3), indicating the translational origin of the diffusion coefficient and the absence of the contribution from the rotational diffusion coefficient to the correlation function. The translational diffusion coefficient was 
then used to calculate the hydrodynamic radii applying the StokesEinstein relation (for more details see Supporting Information).

\section{RESULTS}

We studied the charging and aggregation of TiONWs, which were synthesized by the hydrothermal process as detailed elsewhere, ${ }^{43,46}$ in the presence of an inert salt as well as a polyelectrolyte by electrophoresis and DLS to clarify the stability of the dispersions under different experimental conditions. We have recently published two studies ${ }^{13,14}$ on the dispersion characteristics of aqueous TiONW dispersions, and those results were considered when the experiments were planned, performed, and evaluated in the present work.

Aggregation of Bare Nanowires. As published earlier, ${ }^{13}$ the TiONWs change their charge with the $\mathrm{pH}$, and they are positively charged at low pHs, below the PZC, while their charge becomes negative at higher $\mathrm{pHs}$. This phenomenon is typical for metal oxides including titanium(IV) oxide derivatives due to the protonation-deprotonation equilibrium of the different hydroxyl groups on the surface, and it can be detected by surface potential or EPM measurements. ${ }^{47-51}$ Such charge transition occurred at $\mathrm{pH} 4.1$ in the present system; therefore, the TiONWs were negatively charged in our experiments where the $\mathrm{pH}$ was always set to 9.0. A hydrodynamic radius of $188 \mathrm{~nm}$ was measured by DLS in stable dispersions together with a polydispersity index (PDI, determined by the cumulant analysis in the DLS measurements) of 0.26 , which indicates a relatively narrow particle size distribution (Table 1).

\section{Table 1. Characteristic Dispersion Properties of Bare (TiONW) and Polyelectrolyte Coated (TiONW- PDADMAC) Nanowires ${ }^{a}$}

\begin{tabular}{lccccc}
\multicolumn{1}{c}{ sample } & $\begin{array}{c}R_{\mathrm{h}} \\
(\mathrm{nm})^{b}\end{array}$ & $\mathrm{PDI}^{b}$ & $\begin{array}{c}\mathrm{CCC} \\
(\mathrm{M})\end{array}$ & $\begin{array}{c}\sigma^{c} \\
\left(\mathrm{mC} / \mathrm{m}^{2}\right)\end{array}$ & $\begin{array}{c}\mu^{d} \\
\left(\mathrm{~m}^{2} /(\mathrm{V} \mathrm{s})\right)\end{array}$ \\
TiONW & 188 & 0.26 & 0.008 & -8.2 & -2.3 \\
TiONW- & 202 & 0.34 & 0.032 & +15.3 & +2.5
\end{tabular}

PDADMAC $^{e}$

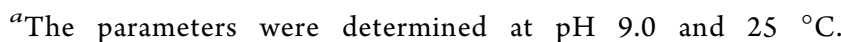
${ }^{b}$ Hydrodynamic radius $\left(R_{\mathrm{h}}\right)$ and polydispersity index (PDI) were determined by DLS in stable dispersions, and the uncertainties of the size measurements were about $5 \mathrm{~nm}$. ${ }^{c}$ Surface charge density $(\sigma)$ was calculated by eq $1 .^{d}$ Electrophoretic mobility $(\mu)$ measured at $0.001 \mathrm{M}$ ionic strength. ${ }^{e}$ Polyelectrolyte dose of $300 \mathrm{mg} / \mathrm{g}$ was used to coat the TiONWs.

DLS measurements revealed that the dispersions were stable at low ionic strengths, but the TiONWs rapidly aggregated at higher electrolyte concentrations. In the latter case at an ionic strength of $0.1 \mathrm{M}$, the increase in the hydrodynamic radius depended on the particle concentration as shown in Figure 1a. Accordingly, the apparent size increased linearly at low concentrations and short time intervals while higher concentration of the nanowires led to saturation-type curves due to the formation of higher generation aggregates. If one compares the slopes obtained at experiment times where the increment was linear in each case, a linear relation can be found with the TiONW concentration (Figure 1a, inset). However, our main goal was to investigate the early stages of the aggregation; therefore, an intermediate concentration of $7 \mathrm{mg} / \mathrm{L} \mathrm{TiONW}$ was chosen for further investigations. Variation of the ionic strength at the same particle concentration gave rise to different slopes in the apparent hydrodynamic radius versus time plots (Figure 1b). No increase in size was found below $0.001 \mathrm{M} \mathrm{KCl}$
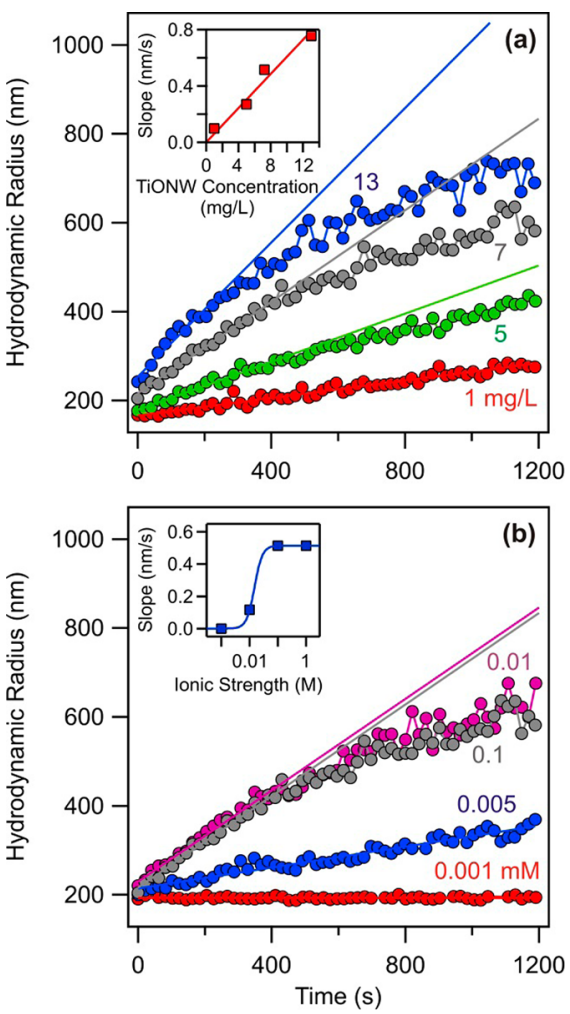

Figure 1. Apparent hydrodynamic radii of TiONWs in time-resolved DLS experiments. (a) Different concentrations of TiONW at $0.1 \mathrm{M}$ ionic strength adjusted by $\mathrm{KCl}$. The inset shows the initial slopes obtained from linear fits at different TiONW concentrations. (b) Aggregation of TiONWs in $7 \mathrm{mg} / \mathrm{L}$ dispersion at different ionic strengths. The initial slopes are presented in the inset as a function of the ionic strength. The experiments were carried out at $25^{\circ} \mathrm{C}$ and $\mathrm{pH}$ 9.0.

concentration, indicating stable dispersions while the slopes increased with the concentration of the electrolyte above this dose, and they remained constant at high ionic strength (Figure $1 b$, inset). Note that only the initial linear regime was considered at high ionic strengths where the aggregation was fast (e.g., at $0.01 \mathrm{M} \mathrm{KCl}$ in Figure $1 \mathrm{~b}$ ), while the whole set of experimental points was used to obtain the slopes at lower salt levels (e.g., $0.005 \mathrm{M} \mathrm{KCl}$ ) during the evaluation of the data.

The stability of the dispersions at different ionic strengths was expressed in terms of the stability ratio (Figure 2). ${ }^{14}$ High stability ratios indicate slow aggregation, i.e., quasi-stable dispersions, while values close to unity correspond to rapidly aggregating, highly unstable samples where the aggregation is exclusively controlled by the diffusion of the particles. Accordingly, the nanowires were stable at low $\mathrm{KCl}$ concentrations and the stability ratios decreased by increasing the ionic strength up to the critical coagulation concentration (CCC) which separates the slow and fast aggregation regimes. The stability ratio values were unity and remained constant at higher electrolyte doses. Linear fits to the two aggregation regimes resulted in a CCC of $0.008 \mathrm{M}$. The EPMs, determined under the same experimental conditions as in the DLS study, increased with the ionic strength but remained negative in the entire concentration range investigated. This result can be explained by the screening effect of the potassium counterions on the surface charge. Moreover, the overall behavior of TiONWs followed the prediction of the theory developed by 




Figure 2. Stability ratio (DLS, squares) and EPM (circles) of TiONWs $(7 \mathrm{mg} / \mathrm{L})$ as a function of the ionic strength adjusted by $\mathrm{KCl}$ at $25^{\circ} \mathrm{C}$ and $\mathrm{pH}$ 9.0. The CCC indicates the position of the critical coagulation concentration, and the lines serve just to guide the eyes.

Derjaguin, Landau, Verwey, and Overbeek (DLVO) for charged colloidal particles in an aqueous electrolyte solution. ${ }^{44}$ Accordingly, the aggregation of the nanowires is governed by the superposition of the repulsive double layer and the attractive van der Waals forces. The electric double layer vanishes at high electrolyte concentrations where the surface charges are completely screened by the counterions leading to the predominance of the attractive van der Waals forces and hence to rapid aggregation of the TiONWs. Similar behavior of the particles was found in other systems containing titanate derivatives and electrolytes. ${ }^{48,49}$ In our previous study, ${ }^{14}$ we have shown by atomic force microscopic images recorded in dispersions that the TiONWs aggregated in a side-by-side orientation leading to a "spaghetti-like" structure of the aggregates. Since the slopes in the fast or so-called diffusion controlled aggregation regime as well as the CCC values were very similar to the values determined for the present system, we assume that the orientation of the TiONWs in the aggregates is the same in both cases.

Tuning the Colloid Stability by Polyelectrolyte. PDADMAC is known as highly charged polyelectrolyte containing quaternary amino groups which are positively charged in water in the entire $\mathrm{pH}$ range. Because of its strong affinity to oppositely charged surfaces, it has been used for modification of negatively charged substrates as well as to tune the aggregation process in aqueous dispersions of colloidal particles. $^{31,34,38}$ In our study, interaction between PDADMAC and the negatively charged TiONW was investigated by following the changes in the EPMs and in the speed of aggregation at different polyelectrolyte doses and ionic strengths.

Figure 3 shows the EPMs in the TiONW-PDADMAC system. In general, the surface charge was constant at very low polyelectrolyte doses, and the initial EPM values were in good agreement with the ones measured in the bare TiONW system at the corresponding ionic strengths. The mobilities increased with increasing the PDADMAC dose due to the strong adsorption of the positively charged polyelectrolyte. Such adsorption led to charge neutralization at the isoelectric point (IEP) where the overall charge of the particles was zero. Upon further addition of PDADMAC to the system, the nanowires reversed their charge, and positively charged particles were obtained beyond the IEP. Such a charge reversal phenomenon is typical in systems containing polyelectrolytes and oppositely charged surfaces ${ }^{21,22,34}$ and can be originated from hydrophobic interactions between the polyelectrolyte chains, ${ }^{52}$ from entropic effect due to the release of solvent and counterions of the highly charged PDADMAC ${ }^{53}$ during its adsorption, and from ion-ion correlations. ${ }^{54,55}$ The dose at the adsorption saturation plateau (ASP) was the limit of the adsorption at high PDADMAC concentrations. At this dose, the surface is not able to adsorb more PDADMAC under the experimental conditions applied and further added polyelectrolytes remained dissolved in the bulk. The EPMs were constant above the dose corresponding to the ASP, but their values on the plateau depended on the ionic strength.

The dependence of the stability ratios on the PDADMAC dose (Figure 3) was found to be similar at lower ionic strengths. Accordingly, the dispersions were stable at low polyelectrolyte concentrations, and the stability ratios decreased until the fast aggregation regime was reached near the IEP. The samples became stable again with increasing the dose after the IEP. These types of colloid stability curves are very typical for
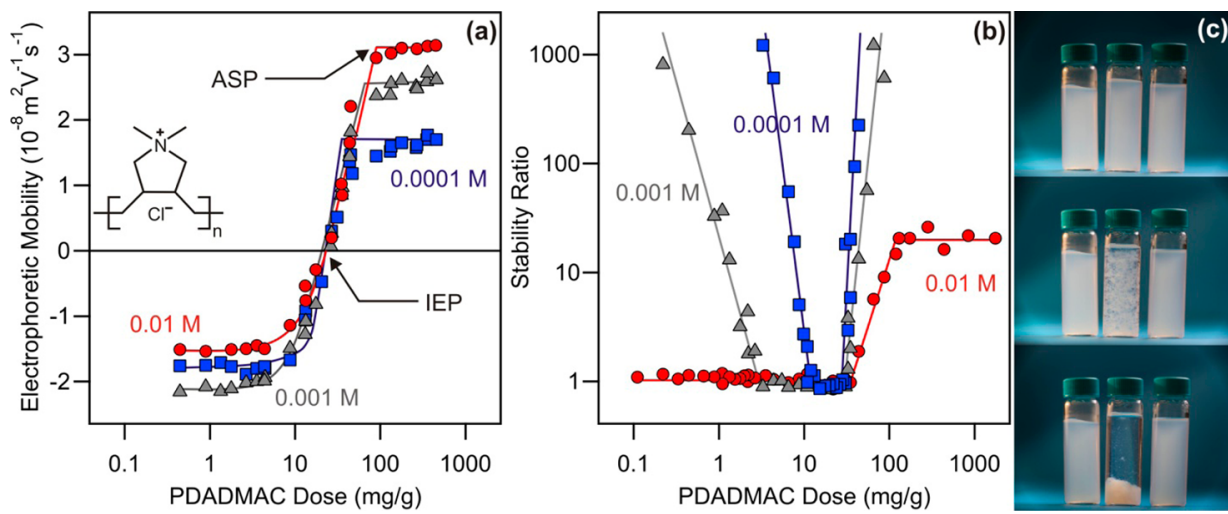

Figure 3. EPM (a) and stability ratio (b) of TiONWs as a function of the PDADMAC dose at different ionic strengths adjusted by $\mathrm{KCl}$ at $25^{\circ} \mathrm{C}, \mathrm{pH}$ 9.0, and TiONW concentration of $7 \mathrm{mg} / \mathrm{L}$. The unit on the $x$-axis denotes $\mathrm{mg}$ of PDADMAC normalized to $1 \mathrm{~g}$ of TiONW. The adsorption saturation plateau (ASP) and the isoelectric point (IEP) are indicated by arrows while the other lines are just to guide the eyes. The chemical structure of PDADMAC is shown in the inset of graph (a). Schematic representation of the colloid stability of TiONW-PDADMAC systems (c). The PDADMAC concentration increased from left to right, while the pictures were taken with freshly mixed samples (top), after 15 min (middle) and $30 \mathrm{~min}$ (bottom). Note that the particle concentration was significantly higher than in the case of aggregation experiments in order to visualize the colloid stability of the systems. 
systems containing colloidal particles and polyelectrolytes, ${ }^{22,33,34}$ and they are in line with the DLVO theory. The TiONWs possessed negative charge at low PDADMAC doses where their charge is only partially neutralized. Such negative charge led to the formation of an electric double layer around the particles and hence repulsive forces between them. Since the nanowires had an overall charge of zero near the IEP, the electric double layer vanished and the attractive van der Waals forces predominated in the dispersion, giving rise to rapid aggregation of the TiONWs and to unstable dispersions. At high doses, the nanowires underwent the charge reversal process due to the PDADMAC adsorption, and the positive charge generated by this phenomenon led to the formation of an electric double layer and to the slowdown of the aggregation due to the overlap of the double layers.

Another interesting issue is the ionic strength dependence of the stability and mobility curves, since we described the interaction forces as of electrostatic origin in the explanation above. If this is the case, change in the concentration of an inert electrolyte, like $\mathrm{KCl}$ in the present case, should have significant effect on the aggregation even in the presence of a polyelectrolyte. $^{21,22}$ Indeed, the shape of the stability ratio versus PDADMAC dose curve at $0.01 \mathrm{M} \mathrm{KCl}$ concentration is different from those measured at lower ionic strengths which were discussed above (Figure $3 \mathrm{~b}$ ). The TiONWs rapidly aggregated at low PDADMAC doses at $0.01 \mathrm{M}$ ionic strength since this salt level is above the CCC value of the bare nanowires (Table 1). However, the aggregation slowed down when the dose around the ASP was approached as indicated by the increase in the stability ratio values which had a plateau at high PDADMAC concentrations, showing the limited stability of the system at this ionic strength.

One can also recognize that the slopes in the stability ratio versus polyelectrolyte dose curves in the slow aggregation regimes were also different at different ionic strengths. Higher $\mathrm{KCl}$ concentration led to less steep curves in this region. We assume that these discrepancies originate from additional, nonDLVO attractions. They are weaker than the DLVO-type forces and can be derived from patch-charge interactions resulting from the lateral heterogeneity of the adsorbed PDADMAC layer. ${ }^{14,22}$ Accordingly, the TiONW surfaces contain negatively charged, uncoated parts at coverage lower than the ASP dose, and they may interact with oppositely charged patches from the adsorbed polyelectrolytes on the surface of another particle. Such patch-charge attraction could contribute to the acceleration of the aggregation of TiONWs and give rise to faster aggregation of the particles, i.e., lower stability ratios, and hence smaller slopes in the slow aggregation regimes which were more sensitive to this additional attraction than the diffusionlimited regime. Since these patch-charge attractions occur at low distances from the surface, they are more pronounced at higher ionic strengths where the Debye length was small and the electric double layers were thin, leading to the emergence of short-range interactions. Another possible interparticle force which was observed in other systems and could be present here as well is the repulsive force due to the osmotic repulsion of the adsorbed PDADMAC chains. ${ }^{35,36,56}$ These forces were found to be repulsive and increased with the ionic strength in the case of monovalent salts. Since in our systems the increase of ionic strength always led to less stable dispersions at the same PDADMAC dose (Figure 3b), the effect of such repulsive interactions should be minor compared to the electrostatic forces (DLVO and patch charge); otherwise, we should have obtained highly stable dispersions at high ionic strength and polyelectrolyte doses.

Additional EPM measurements in the ionic strength range of $0.00001-0.1 \mathrm{M}$ were carried out to study the effect of the inert electrolyte on the IEP and ASP. As shown in Figure 4, the IEP



Figure 4. PDADMAC dose at the isoelectric point (IEP, squares) and at the adsorption saturation plateau (ASP, circles). The data were determined from the EPM versus PDADMAC dose curves at different ionic strengths. The measurements were performed at $25{ }^{\circ} \mathrm{C}, \mathrm{pH} 9.0$ and at a TiONW concentration of $7 \mathrm{mg} / \mathrm{L}$. The solid lines serve only to guide the eyes. The presented values were obtained from linear fits to the experimental data and this method results in an average error of about $5 \%$.

values decreased and the ASP values increased with the ionic strength, indicating that these parameters strongly depend on the $\mathrm{KCl}$ concentration applied. Their magnitudes were similar to those reported in polyelectrolyte adsorption studies previously, and such an increase in the adsorbed amount has been already reported in other systems containing polyelectrolytes and oppositely charged particles. ${ }^{22,57}$ Both phenomena can be explained by the electrostatic interactions between the PDADMAC chains on the surface. Accordingly, the adsorption of the polyelectrolytes was hindered by the electrostatic repulsion between the PDADMAC molecules of the same charge. The increase in the ionic strength, i.e., in the $\mathrm{KCl}$ concentration, led to a screening effect of the polyelectrolyte charge by the chloride ions and also to reduced repulsion between the polyelectrolytes adsorbed on the surface. The TiONWs then were able to adsorb more PDADMAC at higher ionic strength which gave rise to higher ASP and lower IEP values since the polyelectrolyte adsorption is less hindered at higher salt levels.

Aggregation of Polyelectrolyte Coated Nanowires. As discussed earlier, the maximum adsorbed amount of the polyelectrolytes at a certain ionic strength can be expressed with the dose of the ASP, and such adsorption led to an oppositely charged nanowire (TiONW-PDADMAC). Let us discuss now the charging and aggregation of PDADMAC coated TiONWs and compare it to the similar properties of the bare nanowires.

To probe the surface charge and aggregation behavior of the particles and to identify the predominating interparticle forces, the EPMs and stability ratios were measured in dispersions containing TiONWs and PDADMAC at a dose of $300 \mathrm{mg}$ of polyelectrolyte per $1 \mathrm{~g}$ of nanowire (which was higher than the ASP value even at high salt levels) at different ionic strengths (Figure 5). The mobilities of the TiONW-PDADMAC were 


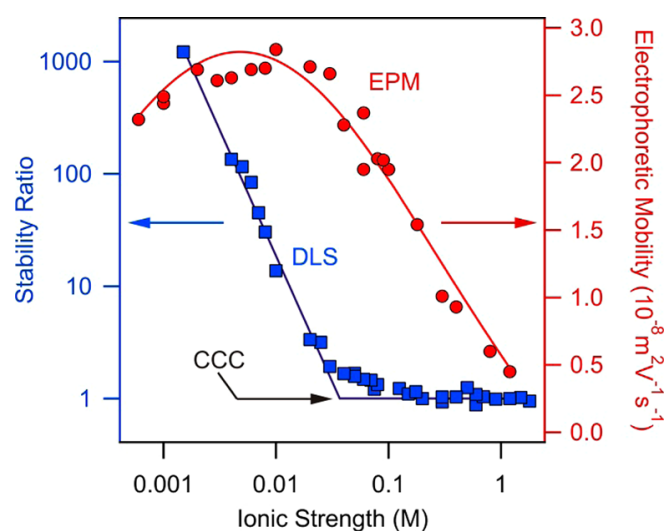

Figure 5. Stability ratio (DLS, squares) and EPM (circles) of TiONW-PDADMAC nanowires (TiONW was coated with PDADMAC at a dose of $300 \mathrm{mg} / \mathrm{g}$ ) as a function of the ionic strength adjusted by $\mathrm{KCl}$ at $25{ }^{\circ} \mathrm{C}$ and $\mathrm{pH}$ 9.0. The TiONW concentration was $7 \mathrm{mg} / \mathrm{L}$. CCC indicates the position of the critical coagulation concentration, and the solid lines serve only to guide the eyes.

positive in the entire ionic strength range investigated. They increased at low electrolyte concentrations and reached a maximum around $0.006 \mathrm{M} \mathrm{KCl}$ concentration. Such maximum was also observed in other systems containing charged colloidal particles and can be originated from ion-ion correlations ${ }^{58}$ and the standard electrokinetic model. ${ }^{59}$ After this maximum, the EPMs decreased with the ionic strength due to the screening effect of the counterions (chloride ions in this case) on the surface. Moreover, the stability ratio values were found to be high at low electrolyte concentrations (Figure 5), indicating the formation of stable dispersions. They decrease with increasing the $\mathrm{KCl}$ concentration until reaching the CCC. Fast aggregation occurred after the CCC and the stability ratios were around unity within the experimental error.

The size of the bare and the coated nanowires determined by DLS in stable dispersions at low ionic strength were close to each other (Table 1), and the small difference can be explained as follows. Upon the preparation of the TiONW-PDADMAC dispersion, the TiONW particles passed through an intermediate stage of polymer adsorption which produced nanowires of zero charge for a very short time interval. During this short period, a part of the TiONWs could aggregate, and hence the apparent hydrodynamic radius slightly increased. This phenomenon was observed in other polyelectrolyte-particle systems, ${ }^{33}$ and the higher PDI value for TiONW-PDADMAC also confirmed this explanation.

Comparing the EPM, diffuse layer potential and stability ratio data obtained in the TiONW and TiONW-PDADMAC systems (Figures 2 and 5, Figures S4 and S5), the following conclusions can be made. The mobility data show the same trend but have the opposite sign due to the charge reversal process. The calculated $\sigma$ data showed that TiONWPDADMAC was almost twice as more charged as the bare TiONWs because the adsorption of the PDADMAC of high line charge density resulted in more charges located at the liquid/solid interface (Table 1). This deviation in $\sigma$ resulted in a significant difference between the CCC values of the bare and coated nanowires. Accordingly, the CCC shifted from 0.008 to $0.032 \mathrm{M}$ after polyelectrolyte coating which gave rise to more stable nanowire dispersions than the original ones without added PDADMAC (Table 1). This is important information and indicates that in the case of polyelectrolyte coating the
TiONW colloids can remain stable at ionic strengths up to 4 times higher than the bare ones for simple monovalent salts.

To further explore the origin of the interparticle forces in the above systems, theoretical calculations were performed to estimate the trend of the stability ratios by the DLVO theory using diffuse layer potentials calculated from the EPMs (Figure S4 and see Supporting Information for more details). The calculations predict also a shift in the CCC values due to the higher surface charge density of the TiONW-PDADMAC particles (Figure S5). The estimated slopes in the slow aggregation regime were very steep and agreed reasonable well with the experimental data for the bare TiONWs, but significant difference was observed in the case of TiONWPDADMAC particles. At very low $\mathrm{KCl}$ concentrations, before the CCC of the theoretical curve, the measured stability ratio values were always much lower than the calculated ones, indicating the presence of additional attractive forces which were not predicted by the DLVO theory. We assume that the previously mentioned patch-charge interactions were responsible for the additional attraction at low ionic strength. Since they are induced by the adsorption of PDADMAC, these forces could not be present in the bare TiONW system; therefore, the agreement between experimental and calculated data was better in that case. However, the transition between the slow and fast aggregation regimes around the $\mathrm{CCC}$ was not sharp in the case of TiONW-PDADMAC compared to the bare TiONW. This deviation could originate from the fact that the adsorbed polyelectrolyte layers usually swell in this ionic strength range, ${ }^{22,33,57}$ resulting in thicker and more hydrated PDADMAC layer on the surface which could lead to steric stabilization of the nanowires due to the osmotic repulsion between the adsorbed polyelectrolyte chains. Such repulsive forces were detected in polyelectrolyte-particle systems at high ionic strength, ${ }^{35,36,56}$ and their effect on the structure of polymer brushes was also described. ${ }^{60}$ Since the stability ratio values were only slightly higher than unity in this regime (Figure S5) and such an effect disappeared at higher ionic strength, where this phenomenon should be more pronounced, we assume that the strength of the osmotic repulsion was small compared to the forces of electrostatic origin.

\section{CONCLUSIONS}

We have studied the surface charge and aggregation of TiONWs in aqueous dispersions in the bare form as well as in the presence of the PDADMAC polyelectrolyte. The bare nanowire dispersions were stable only at low ionic strength at millimolar salt levels. Higher concentration of a monovalent electrolyte led to fast aggregation of the TiONWs and to unstable systems. PDADMAC adsorbed strongly on the oppositely charged TiONW surface, and its adsorption gave rise to charge neutralization at the IEP and subsequent charge reversal at higher polyelectrolyte doses. The adsorption continued until the ASP where the maximum amount of PDADMAC was adsorbed on the surface and further added polyelectrolytes remained dissolved in the solution. Timeresolved DLS measurements performed at different polyelectrolyte doses revealed that the dispersions were unstable near the IEP and stable far before and after it. Adding PDADMAC to the TiONW sample at the dose of ASP resulted in polyelectrolyte coated nanowires. Similar trends were observed in the mobilities and stabilities in the case of both bare and polyelectrolyte coated TiONWs when the ionic strength was varied in these systems. These facts tend to show that the 
aggregation processes were governed by interactions of DLVO origin mainly; however, the presence of attractive patch-charge and repulsive osmotic interactions was also detected especially in the TiONW-PDADMAC system. A highly significant advantage of the nanowire coating with polyelectrolyte was that the PDADMAC coverage resulted in a higher CCC value. In summary, the present study provides quantitative information which allows for designing stable or aggregating dispersions of TiONWs using a polyelectrolyte. Accordingly, the dose of polyelectrolyte, particle concentration, and salt level can be precisely adjusted to tune the dispersion stability in accordance with the desired goals.

\section{ASSOCIATED CONTENT}

\section{S Supporting Information}

Detailed experimental section including materials, light scattering, and DLVO calculations as well as results of static and dynamic light scattering measurements and theoretical calculations. This material is available free of charge via the Internet at http://pubs.acs.org.

\section{AUTHOR INFORMATION}

\section{Corresponding Author}

*E-mail: istvan.szilagyi@unige.ch (I.S.).

\section{Notes}

The authors declare no competing financial interest.

\section{ACKNOWLEDGMENTS}

Financial support from the Swiss National Science Foundation (150162), Swiss Scientific Exchange Program (12029), Swiss Contribution $(\mathrm{SH} / 7 / 2 / 20)$ and the University of Geneva is gratefully acknowledged. T.S., V.T., and I.S. are grateful to Prof. Michal Borkovec for providing access to the light scattering instruments in his laboratory.

\section{REFERENCES}

(1) Koka, A.; Sodano, H. A. High-sensitivity accelerometer composed of ultra-long vertically aligned barium titanate nanowire arrays. Nat. Commun. 2013, 4, 2682-2682.

(2) Horvath, E.; Ribic, P. R.; Hashemi, F.; Forro, L.; Magrez, A. Dye metachromasy on titanate nanowires: sensing humidity with reversible molecular dimerization. J. Mater. Chem. 2012, 22, 8778-8784.

(3) Zhou, W. J.; Liu, X. J.; Sang, Y. H.; Zhao, Z. H.; Zhou, K.; Liu, H.; Chen, S. W. Enhanced performance of layered titanate nanowire-based supercapacitor electrodes by nickel ion exchange. ACS Appl. Mater. Interfaces 2014, 6, 4578-4586.

(4) Hong, Z. S.; Wei, M. D. Layered titanate nanostructures and their derivatives as negative electrode materials for lithium-ion batteries. $J$. Mater. Chem. A 2013, 1, 4403-4414.

(5) Kun, R.; Balazs, M.; Dekany, I. Photooxidation of organic dye molecules on $\mathrm{TiO}_{2}$ and zinc-aluminum layered double hydroxide ultrathin multilayers. Colloids Surf., A 2005, 265, 155-162.

(6) Bajnoczi, E. G.; Balazs, N.; Mogyorosi, K.; Sranko, D. F.; Pap, Z.; Ambrus, Z.; Canton, S. E.; Noren, K.; Kuzmann, E.; Vertes, A.; Homonnay, Z.; Oszko, A.; Palinko, I.; Sipos, P. The influence of the local structure of $\mathrm{Fe}$ (III) on the photocatalytic activity of doped $\mathrm{TiO}_{2}$ photocatalysts-An EXAFS, XPS and Mossbauer spectroscopic study. Appl. Catal., B 2011, 103, 232-239.

(7) Reti, B.; Mogyorosi, K.; Dombi, A.; Hernadi, K. Substrate dependent photocatalytic performance of $\mathrm{TiO}_{2} / \mathrm{MWCNT}$ photocatalysts. Appl. Catal., A 2014, 469, 153-158.

(8) Huang, J. Q.; Cao, Y. G.; Liu, Z. G.; Deng, Z. H.; Wang, W. C. Application of titanate nanoflowers for dye removal: A comparative study with titanate nanotubes and nanowires. Chem. Eng. J. 2012, 191, $38-44$.
(9) Tang, Y. X.; Subramaniam, V. P.; Lau, T. H.; Lai, Y. K.; Gong, D. G.; Kanhere, P. D.; Cheng, Y. H.; Chen, Z.; Dong, Z. L. In situ formation of large-scale $\mathrm{Ag} / \mathrm{AgCl}$ nanoparticles on layered titanate honeycomb by gas phase reaction for visible light degradation of phenol solution. Appl. Catal., B 2011, 106, 577-585.

(10) Toth, M.; Kiss, J.; Oszko, A.; Potari, G.; Laszlo, B.; Erdohelyi, A. Hydrogenation of carbon dioxide on $\mathrm{Rh}, \mathrm{Au}$ and $\mathrm{Au}-\mathrm{Rh}$ bimetallic clusters supported on titanate nanotubes, nanowires and $\mathrm{TiO}_{2}$. Top . Catal. 2012, 55, 747-756.

(11) Potari, G.; Madarasz, D.; Nagy, L.; Laszlo, B.; Sapi, A.; Oszko, A.; Kukovecz, A.; Erdohelyi, A.; Konya, Z.; Kiss, J. Rh-induced support transformation phenomena in titanate nanowire and nanotube catalysts. Langmuir 2013, 29, 3061-3072.

(12) Haspel, H.; Peintler, G.; Kukovecz, Á. Dynamic origin of the surface conduction response in adsorption-induced electrical processes. Chem. Phys. Lett. 2014, 607, 1-4.

(13) Horvath, E.; Szilagyi, I.; Forro, L.; Magrez, A. Probing titanate nanowire surface acidity through methylene blue adsorption in colloidal suspension and on thin films. J. Colloid Interface Sci. 2014, 416, 190-197.

(14) Horváth, E.; Grebikova, L.; Maroni, P.; Szabó, T.; Magrez, A.; Forró, L.; Szilagyi, I. Dispersion characteristics and aggregation in titanate nanowire colloids. ChemPlusChem 2014, 79, 592-600.

(15) Turesson, M.; Nonat, A.; Labbez, C. Stability of negatively charged platelets in calcium-rich anionic copolymer solutions. Langmuir 2014, 30, 6713-6720.

(16) Faraudo, J.; Martin-Molina, A. Competing forces in the interaction of polyelectrolytes with charged interfaces. Curr. Opin. Colloid Interface Sci. 2013, 18, 517-523.

(17) Meszaros, R.; Varga, I.; Gilanyi, T. Adsorption of poly(ethyleneimine) on silica surfaces: Effect of $\mathrm{pH}$ on the reversibility of adsorption. Langmuir 2004, 20, 5026-5029.

(18) Koupanou, E.; Ahualli, S.; Glatter, O.; Delgado, A.; Krumeich, F.; Leontidis, E. Stabilization of lead sulfide nanoparticles by polyamines in aqueous solutions. A structural study of the dispersions. Langmuir 2010, 26, 16909-16920.

(19) Bagaria, H. G.; Yoon, K. Y.; Neilson, B. M.; Cheng, V.; Lee, J. H.; Worthen, A. J.; Xue, Z.; Huh, C.; Bryant, S. L.; Bielawski, C. W.; Johnston, K. P. Stabilization of iron oxide nanoparticles in high sodium and calcium brine at high temperatures with adsorbed sulfonated copolymers. Langmuir 2013, 29, 3195-3206.

(20) Chen, K. L.; Mylon, S. E.; Elimelech, M. Enhanced aggregation of alginate-coated iron oxide (hematite) nanoparticles in the presence of, calcium, strontium and barium cations. Langmuir 2007, 23, 59205928.

(21) Illes, E.; Tombacz, E. The effect of humic acid adsorption on $\mathrm{pH}$-dependent surface charging and aggregation of magnetite nanoparticles. J. Colloid Interface Sci. 2006, 295, 115-123.

(22) Szilagyi, I.; Trefalt, G.; Tiraferri, A.; Maroni, P.; Borkovec, M. Polyelectrolyte adsorption, interparticle forces, and colloidal aggregation. Soft Matter 2014, 10, 2479-2502.

(23) Logar, M.; Bracko, I.; Potocnik, A.; Jancar, B. $\mathrm{Cu}$ and $\mathrm{CuO} /$ titanate nanobelt based network assemblies for enhanced visible light photocatalysis. Langmuir 2014, 30, 4852-4862.

(24) Janaky, C.; de Tacconi, N. R.; Chanmanee, W.; Rajeshwar, K. Bringing conjugated polymers and oxide nanoarchitectures into intimate contact: Light-induced electrodeposition of polypyrrole and polyaniline on nanoporous $\mathrm{WO}_{3}$ or $\mathrm{TiO}_{2}$ nanotube array. J. Phys. Chem. C 2012, 116, 19145-19155.

(25) Munro, C. D.; Plucknett, K. P. Aqueous colloidal characterization and forming of multimodal barium titanate powders. J. Am. Ceram. Soc. 2009, 92, 2537-2543.

(26) Kirby, G. H.; Harris, D. J.; Li, Q.; Lewis, J. A. Poly(acrylic acid)poly (ethylene oxide) comb polymer effects on $\mathrm{BaTiO}_{3}$ nanoparticle suspension stability. J. Am. Ceram. Soc. 2004, 87, 181-186.

(27) Ratinac, K. R.; Standard, O. C.; Bryant, P. J. Lignosulfonate adsorption on and stabilization of lead zirconate titanate in aqueous suspension. J. Colloid Interface Sci. 2004, 273, 442-454. 
(28) Liu, W.; Sun, W. L.; Borthwick, A. G. L.; Ni, J. R. Comparison on aggregation and sedimentation of titanium dioxide, titanate nanotubes and titanate nanotubes- $\mathrm{TiO}_{2}$ : Influence of $\mathrm{pH}$, ionic strength and natural organic matter. Colloids Surf., A 2013, 434, 319-328.

(29) Goyal, N.; Rastogi, D.; Jassal, M.; Agrawal, A. K. Dispersion stabilization of titania nanoparticles for textile: Aggregation behavior and self-cleaning activity. J. Dispersion Sci. Technol. 2013, 34, 611-622.

(30) Hauck, T. S.; Ghazani, A. A.; Chan, W. C. W. Assessing the effect of surface chemistry on gold nanorod uptake, toxicity, and gene expression in mammalian cells. Small 2008, 4, 153-159.

(31) Popa, I.; Cahill, B. P.; Maroni, P.; Papastavrou, G.; Borkovec, M. Thin adsorbed films of a strong cationic polyelectrolyte on silica substrates. J. Colloid Interface Sci. 2007, 309, 28-35.

(32) Xie, F.; Nylander, T.; Piculell, L.; Utsel, S.; Wagberg, L.; Akesson, T.; Forsman, J. Polyelectrolyte adsorption on solid surfaces: Theoretical predictions and experimental measurements. Langmuir 2013, 29, 12421-12431.

(33) Hierrezuelo, J.; Szilagyi, I.; Vaccaro, A.; Borkovec, M. Probing nanometer-thick polyelectrolyte layers adsorbed on oppositely charged particles by dynamic light scattering. Macromolecules 2010, 43, 91089116.

(34) Hierrezuelo, J.; Vaccaro, A.; Borkovec, M. Stability of negatively charged latex particles in the presence of a strong cationic polyelectrolyte at elevated ionic strengths. J. Colloid Interface Sci. 2010, 347, 202-208.

(35) Bauer, D.; Killmann, E.; Jaeger, W. Flocculation and stabilization of colloidal silica by the adsorption of poly-diallyl-dimethylammoniumchloride (PDADMAC) and of copolymers of DADMAC with N-methyl-N-vinyl acetamide (NMVA). Colloid Polym. Sci. 1998, 276, 698-708.

(36) Bauer, D.; Buchhammer, H.; Fuchs, A.; Jaeger, W.; Killmann, E.; Lunkwitz, K.; Rehmet, R.; Schwarz, S. Stability of colloidal silica, sikron and polystyrene latex influenced by the adsorption of polycations of different charge density. Colloids Surf., A 1999, 156, 291-305.

(37) Morga, M.; Adamczyk, Z. Monolayers of cationic polyelectrolytes on mica: Electrokinetic studies. J. Colloid Interface Sci. 2013, 407, 196-204.

(38) Vaccaro, A.; Hierrezuelo, J.; Skarba, M.; Galletto, P.; Kleimann, J.; Borkovec, M. Structure of an adsorbed polyelectrolyte monolayer on oppositely charged colloidal particles. Langmuir 2009, 25, 48644867.

(39) Schaak, R. E.; Mallouk, T. E. Prying apart Ruddlesden-Popper phases: Exfoliation into sheets and nanotubes for assembly of perovskite thin films. Chem. Mater. 2000, 12, 3427-3434.

(40) Zhang, X. T.; Fujishima, A.; Jin, M.; Emeline, A. V.; Murakami, T. Double-layered $\mathrm{TiO}_{2}-\mathrm{SiO}_{2}$ nanostructured films with self-cleaning and antireflective properties. J. Phys. Chem. B 2006, 110, 2514225148.

(41) Ma, R. Z.; Sasaki, T.; Bando, Y. Layer-by-layer assembled multilayer films of titanate nanotubes, Ag- or Au-loaded nanotubes, and nanotubes/nanosheets with polycations. J. Am. Chem. Soc. 2004, 126, 10382-10388.

(42) Mahadeva, S. K.; Walus, K.; Stoeber, B. Piezoelectric paper fabricated via nanostructured barium titanate functionalization of wood cellulose fibers. ACS Appl. Mater. Interfaces 2014, 6, 7547-7553.

(43) Horvath, E.; Kukovecz, A.; Konya, Z.; Kiricsi, I. Hydrothermal conversion of self-assembled titanate nanotubes into nanowires in a revolving autoclave. Chem. Mater. 2007, 19, 927-931.

(44) Trefalt, G.; Szilagyi, I.; Borkovec, M. Poisson-Boltzmann description of interaction forces and aggregation rates involving charged colloidal particles in asymmetric electrolytes. J. Colloid Interface Sci. 2013, 406, 111-120.

(45) Deshiikan, S. R.; Papadopoulos, K. D. Modified Booth equation for the calculation of zeta potential. Colloid Polym. Sci. 1998, 276, 117-124.
(46) Kasuga, T.; Hiramatsu, M.; Hoson, A.; Sekino, T.; Niihara, K. Titania nanotubes prepared by chemical processing. Adv. Mater. 1999, 11, 1307-1311.

(47) Walsh, R. B.; Nelson, A.; Skinner, W. M.; Parsons, D.; Craig, V. $\mathrm{S}$. J. Direct measurement of van der Waals and diffuse double-layer forces between titanium dioxide surfaces produced by atomic layer deposition. J. Phys. Chem. C 2012, 116, 7838-7847.

(48) Snoswell, D. R. E.; Duan, J. M.; Fornasiero, D.; Ralston, J. Colloid stability of synthetic titania and the influence of surface roughness. J. Colloid Interface Sci. 2005, 286, 526-535.

(49) Barringer, E. A.; Bowen, H. K. High-purity, monodisperse $\mathrm{TiO}_{2}$ powders by hydrolysis of titanium tetraethoxide 2 . Aqueous interfacial electrochemistry and dispersion stability. Langmuir 1985, 1, 420-428.

(50) Kuscer, D.; Stavber, G.; Trefalt, G.; Kosec, M. Formulation of an aqueous titania suspension and its patterning with ink-jet printing technology. J. Am. Ceram. Soc. 2012, 95, 487-493.

(51) Niecikowska, A.; Krasowska, M.; Ralston, J.; Malysa, K. Role of surface charge and hydrophobicity in the three-phase contact formation and wetting film stability under dynamic conditions. J. Phys. Chem. C 2012, 116, 3071-3078.

(52) Carrillo, J. Y.; Dobrynin, A. V. Molecular dynamics simulations of polyelectrolyte adsorption. Langmuir 2007, 23, 2472-2482.

(53) Park, S. Y.; Bruinsma, R. F.; Gelbart, W. M. Spontaneous overcharging of macro-ion complexes. Europhys. Lett. 1999, 46, 454460.

(54) Lyklema, J. Overcharging, charge reversal: Chemistry or physics? Colloids Surf., A 2006, 291, 3-12.

(55) Quesada-Perez, M.; Gonzalez-Tovar, E.; Martin-Molina, A.; Lozada-Cassou, M.; Hidalgo-Alvarez, R. Overcharging in colloids: Beyond the Poisson-Boltzmann approach. ChemPhysChem 2003, 4, 235-248.

(56) Fuchs, A.; Killmann, E. Adsorption of polyelectrolytes on colloidal latex particles, electrostatic interactions and stability behaviour. Colloid Polym. Sci. 1998, 279, 53-60.

(57) Seyrek, E.; Hierrezuelo, J.; Sadeghpour, A.; Szilagyi, I.; Borkovec, M. Molecular mass dependence of adsorbed amount and hydrodynamic thickness of polyelectrolyte layers. Phys. Chem. Chem. Phys. 2011, 13, 12716-12719.

(58) Grosberg, A. Y.; Nguyen, T. T.; Shklovskii, B. I. Colloquium: The physics of charge inversion in chemical and biological systems. Rev. Mod. Phys. 2002, 74, 329-345.

(59) Borkovec, M.; Behrens, S. H.; Semmler, M. Observation of the mobility maximum predicted by the standard electrokinetic model for highly charged amidine latex particles. Langmuir 2000, 16, 5209-5212.

(60) Jusufi, A.; Borisov, O.; Ballauff, M. Structure formation in polyelectrolytes induced by multivalent ions. Polymer 2013, 54, 20282035. 